\title{
Aberrant Promoter Hypermethylation of RASSF1a and BRCA1 in Circulating Cell-Free Tumor DNA Serves as a Biomarker of Ovarian Carcinoma
}

\author{
Sandeep Kumar S1, Shalini N Swamy ${ }^{1}$, C S Premalatha², V R Pallavi ${ }^{3}$, Ramesh \\ Gawari ${ }^{1 *}$
}

\begin{abstract}
Objective: Ovarian cancer is one of the leading causes of cancer deaths in women. Ovarian cancer is diagnosed at the late stages and generally relapses within 12-14 months of cytoreductive surgery. This is attributed to lack of precise molecular detection methodologies to detect and track the disease. Epigenetic alteration such as aberrant promoter hypermethylation is an important early event that occurs during cancer development and progression. This study focuses on development of a minimally invasive methylation marker that could be used for detection and prognosis of ovarian cancer patients. Methods: Aberrant promoter hypermethylation of RASSF1a and BRCA1 was assessed in circulating DNA of 72 EOC patients using methylation-specific PCR. The findings were correlated with various clinicopathological parameters. Statistical analysis was done using the Fisher exact test and chi-square test. Results: The aberrant methylation patterns of RASSF1a and BRCA1 was identified to be present in the cancerous samples. A total of $31.9 \%$ and $56.9 \%$ methylation was observed for RASSF1a and BRCA1 respectively. A striking 50\% methylation of $B R C A 1$ was identified in the benign sample cohort, which marks the significance of assessing the hypermethylation pattern to detect cancer at its early stages. Methylation of the two tumor suppressor genes was evident across various stages and grades of ovarian tumors suggesting that this could also help as a prognostic marker. Conclusion: The results of the current study hold significance since the hypermethylation patterns can be identified in the cell-free circulating tumor DNA from a small volume of blood plasma and is a simple and minimally-invasive method. Assessment of hypermethylation patterns of a panel of TSG along with the existing screening markers could aid in better diagnosis and management of the disease. It could also aid in designing specifically tailored treatment strategies to fight the disease.
\end{abstract}

Keywords: Ovarian cancer- promoter hypermethylation- biomarker- circulating tumor DNA- RASSF1a, BRCA1

Asian Pac J Cancer Prev, 20 (10), 3001-3005

\section{Introduction}

Ovarian cancer is the third leading gynecological cancer in India and has a very high mortality rate compared to other cancers (Globacon., 2018). Ovarian cancer is mostly asymptomatic at the early stages and the patients do not report with any major clinical symptoms. The high mortality rate of ovarian cancer is attributed to the diagnosis of the disease at stage III or IV, where the cancer would have already metastasized to distant sites. The five-year survival rate of ovarian cancers diagnosed at stage III and IV is about $42 \%$ and $26 \%$ as against $93 \%$ when it is diagnosed at the early stages (Torre et al., 2018).

Despite advances in diagnosis, it has not been possible to detect the disease at the early stage. There is an increasing need to develop new and novel detection methodologies to identify the disease at the early stages.
The onset of the tumor is attributed to several genetic and epigenetic alterations. One such epigenetic alteration that is known to occur in the early stages of tumor development is the aberrant hypermethylation of the $\mathrm{CpG}$ island in gene promoters. This aberrant hypermethylation is known to cause silencing of several tumor suppressor genes, thereby contributing to malignant transformation. Aberrant hypermethylation of TSGs can be studied in the cell-free circulating DNA of cancer patients.

Detection of molecular changes in liquid biopsies such as peripheral blood is gaining attention due to its advantages over tumor biopsies. Use of peripheral blood to detect molecular changes that occur during cancer progression holds a significant promise as a minimally invasive marker for detection and monitoring of the disease at any stage during therapy.

Cell-free DNA from the tumor cells is shed into the 
bloodstream during the course of tumor progression. Epigenetic alterations such as aberrant promoter hypermethylation patterns can be identified in the cell-free circulating DNA of ovarian cancer patients and therefore, would allow for the real-time monitoring of the disease.

This study aims at studying the aberrant promoter hypermethylation of two tumor suppressor genes, RASSF $1 a$ and BRCA1, known to be involved in ovarian carcinoma pathogenesis.

RAS associated domain family 1 A (RASSF1a), is a tumor suppressor gene located on chromosome $3 \mathrm{p} 21.3$ and is known to be involved in cell cycle regulation. RASSF $1 A$ falls into the category of the genes frequently silenced by methylation rather than mutation events (Chen $\mathrm{H}$ et al., 2006). Aberrant promoter hypermethylation of RASSF la has been reported in various malignancies ranging from a frequency of $99 \%$ in the tumor to $0 \%$ in normal tissues (Donninger et al., 2007).

Breast cancer susceptibility gene 1 ( $B R C A 1)$, is a gene that is located on chromosome 17q12.21 mainly involved in the maintenance of genome stability and effective DNA repair mechanism (Zhang et al.,2015; Koukoura et al., 2014). Effect of genetic and epigenetic alterations in the $B R C A 1$ gene is attributed in many malignancies including breast and ovarian cancers (Esteller et al., 2000). BRCA1 methylation frequencies have been reported to be ranging from 10-89\% in ovarian cancers (Prieske e3et al., 2017).

In the present study, we have assessed the aberrant promoter hypermethylation status of RASSF $1 a$ and $B R C A 1$ in the cell-free circulating DNA (cfc DNA) of ovarian carcinoma patients using methylation-specific PCR.

\section{Materials and Methods}

\section{Patients and sample collection}

Aberrant promoter hypermethylation was assessed in 72 epithelial ovarian carcinoma (EOC) patients and 15 (age matched) healthy subjects. Preoperative blood sample was obtained from all the subjects after obtaining written informed consent. The study has been approved by the Institutional Scientific Review Board and the Medical Ethics Committee.

\section{Blood collection and processing}

$3 \mathrm{ml}$ of pre-operative blood sample was collected from the patients in a BD vacutainer (spray coated K2EDTA tubes). The blood was immediately centrifuged at 3,000 rpm for 10 mins to separate plasma. The plasma samples were stored at $-80^{\circ} \mathrm{C}$ until DNA extraction.

\section{DNA Extraction}

The plasma sample was thawed on ice and incubated with Proteinase K $(10 \mathrm{mg} / \mathrm{ml})$ and $0.5 \%$ SDS overnight at $37^{\circ} \mathrm{C}$. Post incubation the plasma samples were subjected to Phenol: Chloroform extraction and the DNA were precipitated with $10 \mathrm{~mol} / \mathrm{L}$ ammonium acetate and $2 \mu 1$ of glycogen. The precipitated DNA was washed with 70\% ethanol and eluted with $10 \mu \mathrm{l}$ of TE buffer. DNA aliquots were stored at $-20^{\circ} \mathrm{C}$ until bisulfite modification.
Analysis of DNA Methylation by Bisulfite modification

DNA extracted from the plasma samples was subjected to Sodium Bisulfite modification using EZ DNA Methyl Lightening kit TM (Zymo Research, CA, USA) following the manufacturer's protocol. Modified DNA was eluted in a final volume $10 \mu 1$.

Promoter hypermethylation was assessed performing Methylation specific PCR using specific primers adapted from Honorio S et al., (2003) and Esteller $\mathrm{M}$ et al., (2000) (RASSF1A Methylated forward -5'-GGGTTTTGCGAGAGCGCGT-3', RASSF 1A Methylated reverse-5'-GCTAACAAACGCGAACCG-3', RASSF1A Unmethylated forward-5' G G T T T T G T GA GA G T G T G T T TA G T-3', RASSF1A Unmethylated reverse5 ' CACTA ACAAACACA A ACCAAACA - 3'; $B R C A 1 \quad$ M e thylated forward-5' GGGTTTTGCGAGAGCGCGT-3', BRCA1 Methylated reverse-5'-AAAACTCAACGAACTCACGCCG-3', BRCA 1 Unmethylated forward-5' TTGGTTTTTGTGGTAATGGAAAAGTGT-3', BRCA 1 Unmethylated reverse-5' CAAAAAATCTCAACAAACTCACACCA-3'). The cycling conditions are mentioned in Table1.

Universally Methylated HeLa Genomic DNA (New England Biolabs Inc, England) was used as a positive control for the methylated allele. Peripheral blood-derived DNA from healthy non-cancer patients was used as a control for unmethylated allele and no template control served as a negative control. $10 \mu 1$ of the PCR product was loaded on to a $2.5 \%$ Agarose gel and visualized by staining with Ethidium Bromide.

\section{Measurement of CA125 and CEA}

Cancer antigen 125 (CA125) and Carcinoembryonic antigen (CEA) levels were estimated for all the 72 EOC patients and normal subjects included in the study. CA125 and CEA were estimated using the Elecsys CA125 and CEA diagnostic kits procured from Roche, Germany and quantified on Cobas e411TM auto analyzer (Roche Diagnostics, Germany). A CA125 value of $0-35 \mathrm{U} / \mathrm{ml}$ and a CEA value between $0-7.5 \mathrm{ng} / \mathrm{ml}$ were considered normal.

Statistical analysis: The methylation status of RASSF1A and $B R C A 1$ was correlated with clinicopathological characteristics. Chi-square test or Fisher Exact test was used to analyze the data. All the data were statistically analyzed using the SPSS software version 21.0. A p-value of less than 0.05 was considered to hold a statistical significance.

\section{Results}

Methylation frequency of RASSF1a and BRCA1 in $c f c$ $D N A$

Aberrant promoter methylation was assessed in 15 normal samples and 72 tumor samples which included malignant $(n=53)$, low malignant potential tumors $(n=7)$ and benign samples $(\mathrm{n}=12)$.

20 out of $53(38 \%)$ malignant samples, 1 out of the $7(14 \%)$ low malignant potential tumors and 2 of the 12 (17\%) benign samples showed positive for RASSF1a 
DOI:10.31557/APJCP.2019.20.10.3001

Hypermethylation in Circulating DNA of Ovarian Cancer Patients as a Biomarker

Table 1. Methylation Specific PCR Conditions

\begin{tabular}{lccccc}
\hline Gene & Initial Denaturation & \multicolumn{2}{c}{ Cycling Stage $(35$ cycles $)$} & Final Extension \\
& & Denaturation & Annealing & Extension & \\
\hline RASSFla & $95^{\circ} \mathrm{C}$ & $95^{\circ} \mathrm{C}$ & $60^{\circ} \mathrm{C}(\mathrm{M}) / 58^{\circ} \mathrm{C}(\mathrm{UM})$ & $72^{\circ} \mathrm{C}$ & $72^{\circ} \mathrm{C}$ \\
Time & $10 \mathrm{nims}$ & $30 \mathrm{sec}$ & $30 \mathrm{sec}$ & $30 \mathrm{sec}$ & $7 \mathrm{mins}$ \\
$B R C A 1$ & $95^{\circ} \mathrm{C}$ & $95^{\circ} \mathrm{C}$ & $65^{\circ} \mathrm{C}(\mathrm{M}) / 61^{\circ} \mathrm{C}(\mathrm{UM})$ & $72^{\circ} \mathrm{C}$ & $72^{\circ} \mathrm{C}$ \\
Time & $5 \mathrm{mins}$ & $30 \mathrm{sec}$ & $30 \mathrm{sec}$ & $30 \mathrm{sec}$ & $5 \mathrm{~min}$ \\
\hline
\end{tabular}

Table 2. Methylation Frequencies for RASSF1a

\begin{tabular}{lcccc}
\hline Genes & \multicolumn{2}{c}{ Tumor type } & Normal (15) \\
\hline RASSF1A $(\mathrm{M})$ & $20 / 53(38 \%)$ & LMP $(07)$ & Benign $(12)$ & $0 / 15(0 \%)$ \\
RASSF1A(U) & $33 / 53(62 \%)$ & $6 / 7(86 \%)$ & $1 / 12(17 \%)$ & $15 / 15(100 \%)$ \\
$\mathrm{p}$ Value & $0.0033 * *$ & 0.318 & 0.188 & \\
\hline
\end{tabular}

promoter methylation. The data is represented in Table 2. The representative gel image of the same is presented in Figure 1A.

33 of the $53(62 \%)$ malignant tumors, $2 / 7(29 \%)$ low malignant potential tumors and 6 of the $12(50 \%)$ of the benign samples showed positive for $B R C A 1$ promoter methylation. The summary of the same has been represented in Table 3. The representative gel image is presented in Figure 1B.

Methylation in both the genes was observed for 22 samples $(30.5 \%)$ whereas, promoter hypermethylation in either one or both the genes were seen in 42/72 (58.3\%) samples. Neither BRCA1 nor RASSF1A genes showed methylation in the non-cancerous samples.

Presence of both methylated and unmethylated bands were observed in a few samples and this could be due to

Table 3. Methylation Frequencies for BRCA1

\begin{tabular}{lcccc}
\hline Genes & \multicolumn{3}{c}{ Tumor type } & Normal (15) \\
\hline BRCA1 (M) & Malignant (53) & LMP(07) & Benign (12) & $0 / 15(0 \%)$ \\
BRCA1(U) & $33 / 53(62 \%)$ & $2 / 7(29 \%)$ & $6 / 12(50 \%)$ & $15 / 15(100 \%)$ \\
pValue & $20 / 53(38 \%)$ & $5 / 7(71 \%)$ & $6 / 12(50 \%)$ & \\
\hline
\end{tabular}

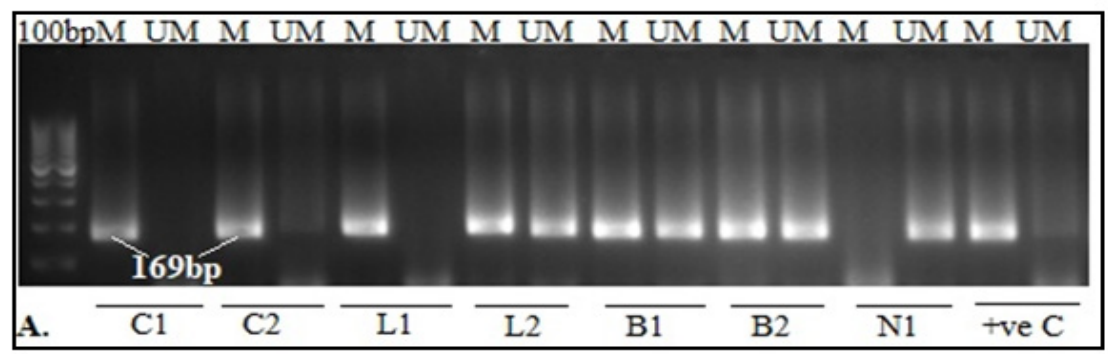

B

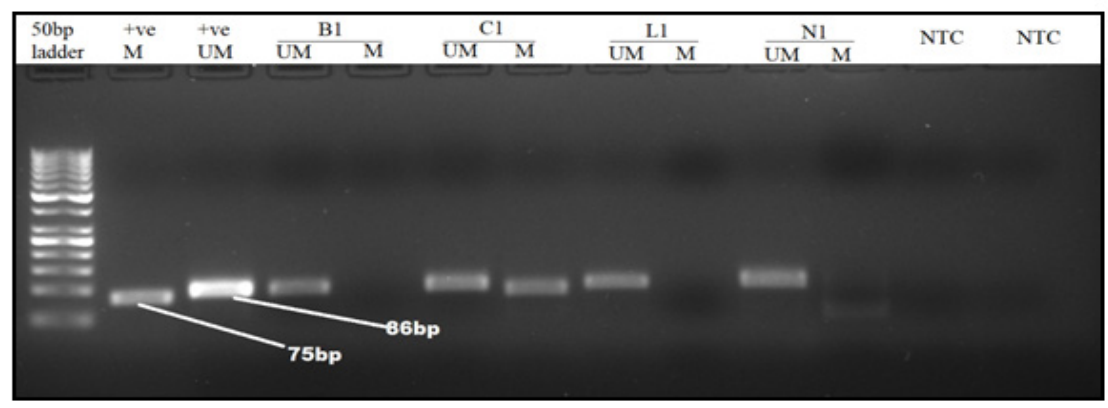

Figure 1. A and B, Representative Agarose gel images of RASSF1a and BRCA1. 2.5\% Agarose gel depicting the products of MSP analysis of (A) RASSF1A and (B) BRCA1 genes in ovarian cancer patients. PCR products in the lanes labeled ' $M$ ' represent the methylated alleles and the lanes labeled 'UM' represent the unmethylated alleles. Universally methylated HeLa genomic DNA was used as a positive (+ve) control and peripheral-blood derived DNA from normal non-carcinoma subjects were used as a positive control for unmethylated alleles. PCR products in lane UM and M indicate the presence of an unmethylated and methylated allele. C1-2(carcinomas), B1-2 (benign adenomas), L1-2 (low malignant potential tumors) and N1 (non-cancer sample). 


\begin{tabular}{|c|c|c|c|c|}
\hline \multicolumn{2}{|l|}{ Clinicopathologic Characteristics } & \multirow{2}{*}{$\begin{array}{l}\mathrm{N} \\
17\end{array}$} & \multirow{2}{*}{$\begin{array}{c}\begin{array}{c}\text { RASSF1A } \\
(\% \text { methylation })\end{array} \\
2 / 17(12 \%)\end{array}$} & \multirow{2}{*}{$\begin{array}{c}\begin{array}{c}\text { BRCA1 } \\
(\% \text { methylation })\end{array} \\
8 / 17(47 \%)\end{array}$} \\
\hline Menopause State $(n=72)$ & Pre Menopause & & & \\
\hline & Post Menopause & 55 & $21 / 55(38 \%)$ & $33 / 55(60 \%)$ \\
\hline & p-Value & & $0.041 *$ & 0.346 \\
\hline \multirow{5}{*}{$\begin{array}{l}\text { Histological type (malignant cohort } \\
\mathrm{n}=53 \text { ) }\end{array}$} & Serous & 36 & $14 / 36(39 \%)$ & $27 / 36(75 \%)$ \\
\hline & Mucinous & 10 & $4 / 10(40 \%)$ & $4 / 10(40 \%)$ \\
\hline & Clear cell & 3 & $1 / 03(33 \%)$ & $1 / 03(33 \%)$ \\
\hline & Endometroid & 4 & $1 / 04(25 \%)$ & $1 / 04(25 \%)$ \\
\hline & p-Value & & 0.952 & $0.042 *$ \\
\hline \multirow[t]{3}{*}{ FIGO Stage $(\mathrm{n}=53)$} & I-II & 15 & $4 / 15(27 \%)$ & $6 / 15(40 \%)$ \\
\hline & III-IV & 38 & $19 / 38(50 \%)$ & $35 / 38(92 \%)$ \\
\hline & p-Value & & 0.123 & $\mathrm{P}<0.001 *$ \\
\hline \multirow[t]{5}{*}{$\mathrm{CA} 125(\mathrm{U} / \mathrm{ml})(\mathrm{n}=72)$} & $0-35$ & 13 & $0 / 13(0 \%)$ & $0 / 13(0 \%)$ \\
\hline & $35-500$ & 16 & $3 / 16(19 \%)$ & $8 / 16(50 \%)$ \\
\hline & $500-1,000$ & 17 & $0 / 17(0 \%)$ & $15 / 17(82 \%)$ \\
\hline & $>1,000$ & 26 & $0 / 26(0 \%)$ & $18 / 26(69 \%)$ \\
\hline & p-Value & & $0.014^{*}$ & $\mathrm{P}<0.001^{*}$ \\
\hline \multirow[t]{3}{*}{ CEA (ng/ml) $(\mathrm{n}=72)$} & $0-7.5$ & 67 & $0 / 67(0 \%)$ & $0 / 67(0 \%)$ \\
\hline & $>7.5$ & 5 & $0 / 5(0 \%)$ & $3 / 5(60 \%)$ \\
\hline & p-Value & & ----- & $<0.001 *$ \\
\hline \multirow[t]{3}{*}{ Ascites $(\mathrm{n}=72)$} & Presence of ascites & 24 & $4 / 24(17 \%)$ & $14 / 24(58 \%)$ \\
\hline & Absence of ascites & 48 & $19 / 48(40 \%)$ & $27 / 48(56 \%)$ \\
\hline & p-Value & & $0.049^{*}$ & 0.866 \\
\hline
\end{tabular}

heterogeneity (presence of both cancer and normal cells). In such a case the samples are considered to be positive for methylation.

\section{Correlations of BRCA1 and RASSF1A Methylation with clinical factors}

All the samples included in this study belonged to the epithelial ovarian carcinoma and they were grouped based on menopausal status, histological subtype, FIGO stage, CA125, CEA levels and the presence or absence of ascites. The details of the same are summarized in table- 3 . Most of the tumors belonged to the high grade and advanced stages $(38 / 53,71.6 \%)$ and the most common histological subtype in the study was of the serous tumors $(36 / 53,67.9 \%)$.

The aberrant hypermethylation status of $B R C A 1$ and RASSF $1 A$ genes were assessed and its association with the available clinicopathological parameters were studied and summarized as in Table 4.

Based on the statistical analysis, a significant correlation was found to exist between the methylation status of $B R C A 1$ with histological type ( $\mathrm{p}=0.042)$, FIGO stages $(\mathrm{p}<0.001), \mathrm{CA}-125(\mathrm{p}<0.001)$ and CEA $(\mathrm{p}<0.001)$ levels. BRCA1 methylation did not show a correlation between the menopausal state and presence of ascites. On the other hand, RASSF1A methylation showed a significant correlation only with the menopausal status $(p=0.041)$ and ascites levels $(p=0.049)$.

\section{Discussion}

Genetic and epigenetic alterations are known to be implicated in the development of several cancers including ovarian cancer. Aberrant promoter hypermethylation is a common epigenetic alteration that causes inactivation of TSGs and is known to be an early event during carcinogenesis (Zuberi et al., 2014). Detection of these epigenetic alterations in cell free circulating DNA in blood and other body fluids is gaining importance due its ease of accessibility and non invasiveness.

In the present study, we have assessed the aberrant promoter hypermethylation of RASSF Ia and BRCAI using methylation specific PCR. A methylation frequency of $56.9 \%$ was observed for $B R C A 1$. Interestingly a $50 \%$ methylation frequency of $B R C A 1$ was observed in the benign sample cohort, suggesting that the methylation of $B R C A 1$ is indeed an early event in ovarian cancer. The methylation frequency of $B R C A 1$ showed a statistically significant correlation with histological subtype, FIGO staging, CA125 and CEA levels. In addition a methylation frequency of $31.9 \%$ was observed for RASSF $1 a$ and was found to be significantly associated with the menopausal status and CA125 levels.

A similar study by De Caceres et al., (2004) have reported a methylation frequency of $18 \%$ for $B R C A 1$ and $42 \%$ for RASSF $1 a$ in the serum of ovarian cancer patients. However there are other studies that have reported the methylation frequency of BRCA1 and RASSF $1 a$ in the range of $16 \%-52 \%$ and $26 \%-66 \%$ respectively (Shilpa 
et al., 2014; Cathy et al.,2005; Bai et al., 2014; Jens et al., 2005; Prakash et al., 2005; Yoon et al., 2001; Bhagat et al., 2012; Matoo et al., 2013). But these finding are derived from the experiments performed using primary tumor tissue specimens and not circulating tumor DNA. Our study is the first report on aberrant hypermethylation of RASSF1A and BRCA1 in cell-free circulating DNA of epithelial ovarian cancer from the Asian population.

The results of the present study and earlier reports are suggestive of the fact that the cell free circulating tumor DNA carries the representative epigenetic signatures of the primary tumor. Identification such aberrant epigenetic alterations that occur during tumorigenesis could thus serve as a promising molecular marker for the early detection of the disease.

In conclusion, Since methylated genes appear to have superior specificity for cancer, highly specific DNA methylation marker of a panel of TSGs can be combined with the existing diagnostic modalities to increase the efficacy in diagnosis of ovarian cancer. Assessment of epigenetic alterations in cell-free DNA of cancer patients can serve as a minimally invasive and potential diagnostic and prognostic marker.

\section{Acknowledgments}

\section{Funding Support}

S.K.S acknowledges the Council of Scientific and Industrial Research for the Senior Research Fellowship award no. 09/999/0002/2016-EMR-I. The research work is supported by Department of Biotechnology, India, Grant No BT/PR 13440/MED/30/267/2009.

\section{References}

Ashraf D, Angelo A, Stella T, et al (2005). Involvement of the RASSF $1 A$ tumor suppressor gene in controlling cell migration. Cancer Res, $65,17$.

Bai X, Fu Y, Xue H, et al (2014). BRCAl promoter hypermethylation in sporadic epithelial ovarian carcinoma: Association with low expression of $B R C A 1$, improved survival and co-expression of DNA methyltransferases. Oncol Lett, 7, 1088-96.

Cathy BW, Bora EB, Holly HG, et al (2005). High-resolution methylation analysis of the $B R C A 1$ promoter in ovarian tumors. Cancer Genet Cytogenet, 159, 114-22.

Chen H, Suzuki M, Nakamura Y, et al (2006). Aberrant methylation of RASGRF2 and RASSF1A in human non-small cell lung cancer. Oncol Rep, 15, 1281-5.

Chiang JW, Karlan BY, Baldwin RL (2006). BRCA1 promoter methylation predicts adverse ovarian cancer prognosis. Gynecol Oncol, 101, 403-10.

De Caceres II, Battagli C, Esteller M, et al (2004). Tumor cell-specific $B R C A 1$ and $R A S S F 1 A$ hypermethylation in serum, plasma, and peritoneal fluid from ovarian cancer patients. Cancer Res, 64, 6476-81.

Donaldson J, Park BH (2018). Circulating tumor DNA: measurement and clinical utility. Annu Rev Med, 69, 223-34.

Donninger H, Vos MD, Clark GJ (2007). The RASSF1A tumor suppressor. J Cell Sci, 120, 3163-72.

Esteller M, Silva JM, Dominguez G, et al (2000). Promoter hypermethylation and $B R C A 1$ inactivation in sporadic breast and ovarian tumors. J Nat Cancer Instit, 92, 564-9.

Giannopoulou L, Chebouti I, Pavlakis K, Kasimir-Bauer S,
Lianidou ES. RASSF1A promoter methylation in high-grade serous ovarian cancer: A direct comparison study in primary tumors, adjacent morphologically tumor cell-free tissues and paired circulating tumor DNA. Oncotarget, 8, 21429.

Globacon (2018). India fact sheet. National Institute of cancer prevention and research. September 2018. http:// cancerindia.org.in/globocan-2018-india-factsheet.

Honorio S, Agathanggelou A, Schuermann M, et al (2003). Detection of RASSF1A aberrant promoter hypermethylation in sputum from chronic smokers and ductal carcinoma in situ from breast cancer patients. Oncogene, 22, 147.

Jens MT, Jacqueline H, Sharon M, et al (2001). Hypermethylation of the $\mathrm{CpG}$ island of The Rassfla gene in ovarian and renal cell carcinomas. Int $J$ Cancer, 94, 212-7.

Koukoura O, Spandidos DA, Daponte A, Sifakis S (2014). DNA methylation profiles in ovarian cancer: implication in diagnosis and therapy. Mol Med Rep, 10, 3-9.

Matoo AA, Khan H, Wani HA, et al (2013). RAS association domain isoform A (RASSF1A) gene hypermethylation in ovarian cancer patients of Kashmir valley. J Pak Med Stud, 3, 87-91.

Min S, Su JS, Nagi GA, et al (2004). The tumour suppressor RASSF $1 A$ regulates mitosis by inhibiting the APC-Cdc20 complex. Nat Cell Biol, 6, 129.

Prakash BM, Saboorian MH, Raheela A, et al (2005). Promoter hypermethylation profile of ovarian epithelial neoplasms. Clin Cancer Res, 11, 5365-9.

Prieske K, Prieske S, Joosse SA, et al (2017). Loss of BRCA1 promotor hypermethylation in recurrent high-grade ovarian cancer. Oncotarget, 8, 83063.

Rahul B, Shilpa C, Premalata CS, et al (2012). Aberrant promoter methylation of the RASSF1A and APC genes in epithelial ovarian carcinoma development. Cell Oncol, 35, 473-9.

Robert B (2005). CpG island methylation of DNA damage response genes in advanced ovarian cancer. Cancer Res, 19, 8961-7.

Rohini R, Jarin C, Simon N (2012). Powell. BRCA1 and BRCA2: different roles in a common pathway of genome protection. Nat Rev, 1, 68.

Shairaz B, Stella T, Sarah F, et al (2005). The tumor suppressor RASSF $1 A$ and MAP-1 link death receptor signaling to bax conformational change and cell death. Mol Cell, 2005, 637-50.

Shilpa V, Rahul B, Premalata CS, et al (2014). BRCA1 promoter hypermethylation and protein expression in ovarian carcinoma-an Indian study. Tumor Biol, 5, 4277-84.

Torre LA, Trabert B, DeSantis CE, et al (2018). Ovarian cancer statistics, 2018. CA Cancer J Clin, 68, 284-96.

Yazici H, Terry MB, Cho YH, et al (2009). Aberrant methylation of RASSF $1 A$ in plasma DNA before breast cancer diagnosis in the Breast Cancer Family Registry. Cancer Epidemiol Prev Biomarkers, 18, 2723-5.

Zhang L, Long X (2015). Association of BRCA1 promoter methylation with sporadic breast cancers: Evidence from 40 studies. Sci Rep, 5, 17869.

Zuberi M, Mir R, Dholariya S, et al (2014) RASSF1 and PTEN promoter hypermethylation influences the outcome in epithelial ovarian cancer. Clin Ovarian Cancer Other Gynecol, 7, 33-9.

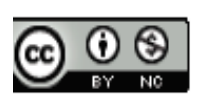

This work is licensed under a Creative Commons AttributionNon Commercial 4.0 International License. 